\title{
Potential liability of reproductive endocrinologists for high order multiple gestation
}

\author{
Mostafa Borahay $\cdot$ John Phelps
}

Received: 7 December 2009 / Accepted: 5 February 2010/Published online: 2 March 2010

(C) US Government 2010

\begin{abstract}
Background In light of the recent octuplet birth and the accompanying intensive media coverage, there has been much attention on high order multiple births resulting from assisted reproductive technology.

Objectives The purpose of this commentary is to review 1) the relative contribution of ART to high order multiple gestation and its impact on infant morbidity, mortality, and health care dollar loss; 2) American Society of Reproductive Medicine's guidelines for the number of embryos transferred in ART; and 3) how reproductive endocrinologists can lessen their exposure to litigation by following the ASRM guidelines for the number of embryos transferred and documenting proper informed consent in the medical records.

Recommendations In situations in which the number of embryos transferred is in excess of the ASRM guidelines, justification for deviating from the ASRM guidelines should be justifiable and documented in the medical records.
\end{abstract}

Capsule Reproductive endocrinologists can lessen their potential exposure to litigation by following the ASRM guidelines on the number of embryos transferred in ART and providing proper informed consent.

\footnotetext{
M. Borahay $(\bowtie) \cdot J$. Phelps

Department of Obstetrics \& Gynecology,

University of Texas Medical Branch,

301 University Blvd,

Galveston, TX 77555-0587, USA

e-mail: maboraha@utmb.edu

M. Borahay

Department of Obstetrics \& Gynecology, Faculty of Medicine,

Zagazig University,

Zagazig, Egypt
}

Keywords High order multiple births · Infertility · Informed consent $\cdot$ IVF $\cdot$ Liability

\section{Introduction}

Assisted reproductive technology (ART) has been associated with a 30 -fold increase in multiple pregnancies compared with the rate of spontaneous twin pregnancies $(1 \%$ in the general white population) [1]. In 2006, the U.S. rate of multiple pregnancies in women undergoing ART was $31.8 \%$, while the percentage of those with high order multiple pregnancies (triplets or more) was 3.8\% [2]. In 2004, ART was responsible for 49,376 out of 4,112,052 total births (1.2\%), 22,226 out of 132,219 twin births (16.8\%), and 2,928 out of $7,275(40.2 \%)$ of high order births [3].

The obstetric and neonatal risks associated with multiple gestations include pre-eclampsia, gestational diabetes, preterm delivery, low birth weight, and operative delivery. Multifetal births account for $17 \%$ of all preterm births (before 37 weeks of gestation), 23\% of early preterm births (before 32 weeks of gestation), 24\% of low-birth weight infants $(<2,500 \mathrm{~g})$, and $26 \%$ of very low birth weight infants $(<1,500 \mathrm{~g})$ [1]. With higher number of fetuses, the average length of gestation decreases. For example, the average lengths of gestation for singleton, twins, triplets and quadruplets fetuses are 39 weeks, 35 weeks, 33 weeks and 29 weeks, respectively.

The incidence of severe handicap in neonatal survivors of multiple gestation is increased from 19.7 per 1,000 singleton survivors to 34 and 57.5 per 1,000 twin and triplet survivors, respectively [4]. These severely handicapped children are likely to continue to require custodial care into their adulthood. The requirements for prolonged health care in these cases can devastate a couple both financially and 
emotionally, especially when a couple has to provide care for multiple handicapped children. The March of Dimes estimated that in 2001, charges for hospital stays for infants with any diagnosis of prematurity were approximately $\$ 13.6$ billion or about $50 \%$ of all birth-related spending. The Institute of Medicine estimated that the societal economic burden associated with preterm birth in the United States in 2005 was at least $\$ 26.2$ billion, or $\$ 51,600$ per infant born preterm [5].

\section{American Society of Reproductive Medicine and Society of Assisted Reproductive Technology practice guidelines on the number of embryos transferred}

In November 1996, the Practice Committee of the Society of Assisted Reproductive Technology (SART), concerned about the number of multiple births due to ART, developed guidelines for the number of embryos to be transferred, based on patient age and number of previous unsuccessful ART cycles. The first of these guidelines was published in 1998 while the most recent [6] was published in November 2008 (summarized in Table 1) to assist ART programs and patients in determining the appropriate number of cleavagestage (usually 2 days or 3 days after fertilization) embryos or blastocysts (usually 5 days or 6 days after fertilization) to transfer. As a result of the application of these guidelines, the ratio of high order multiple births to total ART births decreased substantially since 1998. However, U.S. high order multiple births did not decrease due to a $67 \%$ increase in total ART births and an increase in triplet births from ovulation induction [3].

Recently, lawmakers in certain states (Georgia and Missouri) are studying bills to limit the number of transferred embryos in ART [7]. Some countries (eg, UK, Belgium, and Sweden) have enacted laws to reduce the number of transferred embryos. For example, in an effort to prevent

Table 1 American Society for Reproductive Medicine recommended limits on the numbers of embryos to transfer

\begin{tabular}{lllll}
\hline Prognosis & Age $<35$ & Age 35-37 & Age 38-40 & Age $>40$ \\
\hline Cleavage-stage embryos $^{2}$ & & & \\
Favorable $^{\mathrm{a}}$ & $1-2$ & 2 & 3 & 5 \\
All others & 2 & 3 & 4 & 5 \\
Blastocysts & 1 & 2 & 2 & 3 \\
Favorable $^{\mathrm{a}}$ & 1 & 2 & 3 & 3 \\
All others & 2 & & & \\
\hline
\end{tabular}

${ }^{\mathrm{a}}$ Favorable $=$ First cycle of IVF, good embryo quality, excess embryos available for cryopreservation, or previous successful IVF cycle. Adapted from: Guidelines on number of embryos transferred. Practice Committee of Society for Assisted Reproductive Technology; Practice Committee of American Society for Reproductive Medicine. Fertil Steril 2009;92:1518-9. Epub 2009 Oct [6] high order gestations and to reduce the number of twin gestations, the U.K. Human Fertilization and Embryology Authority (HFEA) legislation limited the number of embryos that can be transferred during a single ART cycle to a maximum of 2 for women under 40 years and 3 for women 40 years and over. Despite a continuing (but slower) upward trend in twin maternities, analysis of data showed that the rate of triplet and higher order births in England and Wales has declined by one-quarter since 1998 [8]. These strict limitations do not allow treatment plans to be individualized after careful consideration of each patient's own unique circumstances. In contrast to these countries, the United States currently doesn't have federal or state laws governing the number of transferred embryos. In an effort to decrease the number of multiple gestations from ART, the ASRM published guidelines for the number of embryos transferred. As guidelines, the ASRM guidelines allow for discretion by reproductive endocrinologists in determining the appropriate number of embryos to transfer. This number may be modified according to individual clinical conditions, including patient age, embryo quality, the opportunity for cryopreservation, and the accumulation of clinical experience with newer techniques.

\section{Potential liability of reproductive endocrinologists}

Reproductive Endocrinologists and their IVF clinics could potentially be held legally accountable for the premature births resulting from the transfer of an excessive number of embryos. There are possibly two separate but intertwined potential causes of action. The first is medical negligence for transferring embryos in excess of the ASRM guidelines without sound justification. The second is failure to obtain informed consent. To have a cause of action for medical negligence for transferring an excessive number of embryos, the patient will have to prove by the preponderance of evidence that 1) the reproductive endocrinologist breached the acceptable standard of medical care by transferring an excessive number of embryos and 2) the breach in the acceptable standard of medical care was the proximate cause of injury to the patient and/or her offspring derived from IVF treatment. For example, in a situation in which the reproductive endocrinologist deviates from the ASRM guidelines without medical justification and this deviation results in a high order multiple gestation, the parents and the offspring have a reasonable cause of action against the reproductive endocrinologist and the IVF facility. Once the burden of proving a breach of the standard is met, couples faced with raising multiple infants with severe handicaps caused by premature delivery could seek an array of damage compensations. Actual economic damages would include the health costs associated with premature delivery, raising 
severely handicapped children, and lifetime custodial care. Economic damages alone for the rearing of multiple preterm infants could easily amount to millions of dollars. In addition to economic damages, damages for pain and suffering may also be awarded. If it is proven that the reproductive endocrinologist acted recklessly with conscious indifference to the safety of his patient and the potential offspring by transferring an excessive number of embryos, punitive damages could also be awarded. Even in states that have tort reform (e.g. Texas, Oklahoma, Colorado ...), punitive damages are usually not capped.

An alternative cause of action is failure to provide informed consent. There are two standards for failure to obtain informed consent: the prudent physician standard and the reasonable patient standard. The standard applied will depend on the jurisdiction in which the lawsuit is being litigated. The prudent physician standard is the majority standard applicable in most states [9]. The prudent physician standard is what a reasonably prudent physician would have disclosed to a patient under similar circumstances. This requires expert testimony by a physician as to what a prudent physician should disclose to a patient. The reasonable patient standard requires the physician to disclose those risks a reasonable patient would want to know. This would include informing the patient of the risks of multiple gestation. The reasonable patient standard is the minority standard, and unlike the prudent physician, does not require expert testimony. To lessen exposure to litigation from failure to provide proper informed consent, the newly published SART informed consent template for IVF is recommended [10]. The consent template is comprehensive and includes risks to patients undergoing IVF as well as their offspring. Multifetal gestation is documented in detail along with other risks including low birth weight and birth defects. This template provides an excellent platform to help patients make an adequately informed consent and, at the same time, lessen the exposure of reproductive endocrinologists and fertility centers to possible litigation.

Once the patient signs the consent, it is a rebuttable presumption that proper informed consent was obtained. Nevertheless, this is a "rebuttable" presumption, and a patient who files a lawsuit will have an opportunity to rebut the presumption that proper informed consent was obtained. For this reason, proper documentation in the medical records is crucial. It is also important to be cognizant that informed consent should not be delegated to nurses or other clinic personnel [11] and that the reproductive endocrinologist is the only one with the duty to provide informed consent to the patient.
In summary, we recommend adherence to the American Society of Reproductive Medicine guidelines for the number of transferred embryos in assisted reproductive technology. Any departure from these guidelines should be justifiable and documented in the medical records. It is crucial not only to providing quality patient care, but also to lessen exposure to liability, to provide proper informed consent and document informed consent in the medical records. We encourage use of the new SART IVF consent template as a foundation on which to counsel patients and provide informed consent. In the US, ART has been selfregulated so far. Cases of unjustified deviation from ASRM guidelines may draw outside regulation that might not be in the patients' best interests.

\section{References}

1. Perinatal risks associated with assisted reproductive technology. ACOG Committee Opinion No. 324. American College of Obstetricians and Gynecologists. Obset Gynecol 2005;106:1143-6.

2. Centers for Disease Control and Prevention. Assisted Reproductive. 2006. Technology (ART) Report: National Summary. Atlanta, GA: US Dept of Health and Human Services. Available at: http://www.cdc.gov/ART/ART2006/index.htm.

3. Dickey R. The relative contribution of assisted reproductive technologies and ovulation induction to multiple births in the United States 5 years after the Society for Assisted Reproductive Technology/American Society for Reproductive Medicine recommendation to limit the number of embryos transferred. Fertil Steril. 2007;88:1554-61.

4. Luke B, Keith LG. The contribution of singletons, twins and triplets to low birth weight, infant mortality and handicap in the United States. J Reprod Med. 1992;37:661-6.

5. Armstrong J. 17 Progesterone for preterm birth prevention: a potential 2 billion dollar opportunity. Am J Obstet Gynecol. 2007;196:194-5.

6. Guidelines on number of embryos transferred. Practice Committee of Society for Assisted Reproductive Technology; Practice Committee of American Society for Reproductive Medicine. Fertil Steril 2009;92:1518-9. Epub 2009 Oct

7. Mccaffrey S: 'Octomom' spawns bills limiting embryo implants. Associated Press. March 4, 2009. Available at: http://www.ap.org.

8. Simmons R, Doyle P, Maconochie N. Dramatic reduction in triplet and higher order births in England and Wales. BJOG. 2004;111:856-8.

9. Kapp M. Patient autonomy in the age of consumer-driven health care: informed consent and informed choice. J Health \& Biomed Law. 2006;1:1-31.

10. Template of informed consent for assisted reproduction. Society of Assisted Reproductive Technology informed consent subcommittee. December 2008. Available at SART website, member only section at www.sart.org.

11. Informed Consent. American Medical Association. Available at AMA website at: http://www.ama-assn.org/ama/pub/physician-resources/ legal-topics/patient-physician-relationship-topics/informed-consent. shtml. 